\title{
Grande vitesse ferroviaire, concurrence et territoires
}

\author{
Antoine Frémont \\ Directeur de recherche \\ Université Gustave Eiffel \\ antoine.fremont@univ-eiffel.fr
}

\section{Pour citer cet article :}

Frémont Antoine, (2019), Grande vitesse ferroviaire, concurrence et territoires, $L$ 'Espace

géographique, $\mathrm{n}^{\circ} 4$, Tome 48, pp. 350-358.

Article disponible en ligne à l'adresse : https://www.cairn.info/revue-espace-geographique-2019-4-page-350.htm

\section{Résumé}

La concurrence appliquée à la grande vitesse ferroviaire est sans doute bénéfique pour accroitre les performances des opérateurs de transport, comme le montre l'article de F. Antoniazzi et al (2019). Mais l'est-elle pour les territoires ? L'article analyse alors les motivations et intérêts de quatre acteurs différents par rapport à la grande vitesse ferroviaire: les voyageurs, les transporteurs, le gestionnaire de réseau et les territoires. Notre hypothèse principale est que la concurrence renforce la dynamique propre des réseaux qui favorise les pôles, le débit et la massification dans une logique de métropolisation. Elle accentue par conséquent les contrastes territoriaux. Les territoires, eux-mêmes en concurrence, permettent d'amortir à la marge l'effet d'archipel issu des réseaux à grande vitesse. Entre le temps court de la concurrence et celui plus long de la cohésion territoriale, les formes de la régulation entre les différentes parties prenantes restent à inventer.

\section{Mots clés}

Grande vitesse ferroviaire, TGV, LGV, territoire, réseau, concurrence, inégalités territoriales

\begin{abstract}
The paper by F. Antoniazzi and al. (2019) highlights the benefit of competition to increase the performance of rail operators engaged in the high speed rail market. But what are the consequences of this competition for cities and the whole country? Our aim is to analyse the motivations and interests of four different players dealing with the high speed market: the passengers, the railway companies, the railway infrastructure manager and the public authorities, in particular the French State and the local governments. Our main hypothesis is that competition reinforces the dynamics of networks based on interconnection of large nodes, high-capacity routes and massification. Therefore it strenghtens spatial disparities. The local governments are themselves in competition in their attempts to attract high speed line investments with as a result the tremendous reduction of travel time between Paris and the other major French cities. They cushion at the margin the consequences of competition. The quick impacts of competition are in contradiction with the need for long-term to foster territorial cohesion. New forms of regulation need to be imagined to overpass this contradiction.
\end{abstract}

\section{Key words}

High speed rail, High speed line, network, local government, competition, spatial disparities 


\section{Introduction}

La littérature sur les effets structurants des infrastructures de transport et notamment sur la grande vitesse ferroviaire (Bazin et al., 2010) est très abondante. Elle ne fait que confirmer et affiner les principales conclusions auxquelles étaient déjà arrivé François Plassard dans les années 1980 et 1990 : " les réseaux n'ont pas d'effets automatiques, ils peuvent donner naissance à de nouvelles stratégies spatiales, ils amplifient les tendances existantes » (Plassard, 1992).

Tout l'intérêt de l'article proposé par F. Antoniazzi et al. (2019) réside dans son parti pris. Loin des effets structurants, l'article se place du point de vue du transporteur ferroviaire et plus particulièrement d'un nouvel entrant sur le marché italien de la grande vitesse ferroviaire qui agit en fonction de sa logique propre et de ses intérêts: conquérir des parts de marché pour gagner de l'argent dans le cadre concurrentiel du grand marché européen voulu par l'Union européenne. Les auteurs démontrent alors aisément que la concurrence est vertueuse parce qu'elle permet de diminuer les coûts des transporteurs, ce qui fait gagner collectivement en efficacité.

Cette conclusion appelle réponse car elle néglige d'évaluer les conséquences de cette concurrence pour les territoires. Afin d'esquisser des pistes d'évaluation, nous reprenons le parti pris de l'article en nous plaçant du point de vue des différents acteurs, en tentant de comprendre leurs motivations et leurs intérêts, mais en confrontant les logiques de quatre acteurs différents: les voyageurs, les transporteurs, le gestionnaire de réseau et les territoires. Quelles sont leurs stratégies dans un contexte concurrentiel, et comme il est question de transport, quelles sont leurs stratégies par rapport aux lieux et aux liens qui les unissent grâce aux réseaux ? Notre hypothèse principale est alors que la concurrence renforce la dynamique propre des réseaux qui accentue elle-même les contrastes territoriaux.

\section{Les voyageurs}

Pour les voyageurs, le TGV incarne une nouvelle modernité, attractivité et compétitivité du train par rapport aux autres modes de transport. De nombreuses études et statistiques le prouvent : le TGV est imbattable par rapport à la voiture individuelle et à l'avion sur des trajets de centre-ville à centre-ville pour des temps de parcours ferroviaires compris entre 1 heure et 3 heures. II réduit de moitié, voire plus, le temps de trajet par rapport à la voiture alors qu'un train classique ne propose que des performances assez proches de cette dernière. En porte à porte, il est aussi rapide voire plus que l'avion sur des distances de 500 à 600 kilomètres. Les parts de marché de l'avion s'effondrent d'ailleurs avec la mise en service d'une LGV sur de telles liaisons (Paris-Marseille, Paris-Bordeaux, ParisStrasbourg) à tel point que la pérennité des liaisons aériennes peut se poser et qu'il est donc relativement aisé d'en annoncer la suppression au service de l'impératif écologique.

Le TGV permet des allers-retours dans la journée de centre à centre dans de bonnes conditions de confort et en toute sécurité, tout en permettant une journée de travail, il est vrai au prix le plus souvent d'une grosse fatigue, d'un point à un autre, avec aux deux bouts un métro ou au mieux une petite marche. Il offre un tunnel d'une salle de réunion à l'autre. La grande vitesse ferroviaire vise d'abord une clientèle relativement limitée symbolisée par les cadres qui voyagent aux frais de leur entreprise. La démocratisation de la grande vitesse ferroviaire n'est possible qu'à travers des prix ajustés en fonction de l'offre et de la demande (yield management) et qui permettent d'offrir en heures creuses des tarifs bas afin d'assurer le remplissage des trains. 
Ce n'est donc pas la concurrence entre les transporteurs ferroviaires qui explique la compétitivité retrouvée du train mais bien une offre ferroviaire entièrement renouvelée depuis les années 1980 .

\section{Les transporteurs}

L'objectif du transporteur ferroviaire est simple, assez similaire à celui de tous les transporteurs : remplir ses véhicules (ici des trains), en assurer la rotation la plus rapide possible, mettre en œuvre les économies d'échelle grâce à des capacités de transport toujours plus élevées (TGV doubles rames et deux étages). Un service ferroviaire à grande vitesse atteint sa rentabilité maximale lorsqu'il relie deux grandes villes distantes de 200 à 800 kilomètres sans effet frontière, le temps que la grande vitesse ferroviaire produise ses effets par rapport à la voiture et qu'ils ne se dissipent pas par rapport à l'avion qui reprend l'avantage sur la très grande distance. Les lois de la gravité ${ }^{1}$ assurent des flux massifs entre les deux points. Ils prennent encore de l'ampleur avec une distance-temps raccourcie, ce que mettent en exergue les études socio-économiques basées principalement sur la valeur du temps permettant ainsi de justifier économiquement de tels services (Quinet, 2013).

De ce point de vue, la géographie centralisée de la France est presque conçue pour le TGV ! Les liaisons en radiales entre Paris et les plus grandes villes de province sont les seules liaisons avec une véritable rationalité économique aux yeux du transporteur. Un réseau optimum se limiterait à quelques liaisons directes, principalement Paris-Lyon, Paris-Marseille, Paris-Bordeaux, Paris-Nantes, Paris-Strasbourg mais aussi, malgré la frontière, Paris-Bruxelles ou Paris-Londres et si possible un Paris-Toulouse en trois heures contre quatre actuellement.

Mais malheureusement pour le transporteur, la géographie des villes françaises est plus complexe. Des villes de second rang se situent en position intermédiaire sur ces liaisons principales! S'y arrêter indirectement grâce à une gare TGV remet en cause le modèle des minutes gagnées : un arrêt d'un TGV sur une LGV se solde par une petite vingtaine de minutes perdues par rapport à un trajet direct ! Les desservir directement oblige à des détours et à prendre le risque de faibles coefficients de remplissage. Au transporteur de trouver les compromis entre l'exigence de la desserte voulue par les voyageurs représentés par leurs associations et leurs élus et la rationalité du transport.

Prenons l'exemple des TGV dits inter-secteurs, qui relient deux grandes villes de province sans passer par Paris. Ils peuvent être presque assimilés à des services radiaux déguisés qui se vident et se remplissent dans les gares périphériques de l'lle-de-France, à Roissy, Marne-la-Vallée ou Massy, et qui cabotent dans les gares intermédiaires afin d'assurer leur remplissage, la valeur du temps entre les deux points extrêmes reliés en ayant alors moins qu'entre ces derniers et les gares franciliennes.

Pour le transporteur, l'équation s'avère encore plus difficile dès qu'il s'agit d'aller desservir des périphéries. La Bretagne (qui n'est en rien une périphérie tant elle est aujourd'hui attractive mais qui en est bien une pour les réseaux car elle reste une péninsule) est un bon cas d'école. Un Paris-Brest ou un Paris-Quimper en tout TGV avec des arrêts multiples à partir de Rennes est une pure aberration pour le transporteur. Le TGV qui se vide largement à Rennes devient de fait ensuite un simple TER sur voie classique sauf que le coût d'exploitation d'un TGV est bien plus élevé que celui d'une rame TER.

Le TGV et plus précisément les quelques liaisons radiales principales jouent un rôle primordial dans l'équilibre économique de la SNCF (cf. Encadré 1). Dans un cadre concurrentiel et du fait d'un coût

\footnotetext{
${ }^{1}$ Le modèle gravitaire de Newton a été transféré à la géographie des transports. Les masses des lieux représentent les facteurs d'émissivité et d'attractivité qui produisent l'interaction alors que son intensité est inversement proportionnelle à la valeur de la distance séparant les lieux » (Giuseppe Pini,1992)
} 
d'entrée élevé (achat du matériel et commercialisation), un opérateur concurrent à l'opérateur historique irait se placer très naturellement sur les segments les plus rentables. C'est précisément la stratégie jouée en Italie par la compagnie Nuovo Trasporto Viaggiatori (NTV) avec le soutien de la SNCF! Le seul axe veritable qui compte relie "I'Italie utile", de Turin à Rome en passant par Milan et Bologne. L'Espagne pourrait être réduite à Madrid-Barcelone, l'Angleterre à Londres-BirminghamManchester, la Pologne à deux axes (Varsovie à Wroclaw et Varsovie à Cracovie)... L'Allemagne est plus compliquée pour la grande vitesse ferroviaire avec son tissu dense de grandes villes notamment en Allemagne rhénane. Mais les liaisons entre ces dernières et Berlin s'imposent depuis que cette dernière est redevenue capitale en 1990.

Les leçons qu'il est possible de tirer de la concurrence qui existe dans le fret ferroviaire en France depuis 2003 (pour l'international) et 2006 (pour le transport intérieur) sont similaires. Les concurrents à l'opérateur historique sont installés sur les axes de trafics les plus importants. Leur part de marché s'élève désormais à 30\% dans le cadre d'un marché ferroviaire qui, loin de se développer sous l'effet de la concurrence, s'est rétracté en raison des évolutions structurelles de l'industrie française. Par contre, et comme le montre l'article de F. Antoniazzi et al. (2019) sur le transporteur NTV en Italie, ils ont permis de réduire les coûts. Pour leur résister, la SNCF a créé sa propre filiale fret « low cost »VFLI. Elle le fait d'une façon similaire et innovante avec l'offre TGV Ouigo afin d'offrir un transport à très grande vitesse aux plus bas coûts possibles et, par répercussion, aux plus petits prix possibles, TGV Ouigo qu'elle s'apprête à lancer en Espagne sur les trois liaisons principales entre Madrid et Barcelone, Madrid et Valence, Madrid et Séville.

La concurrence en matière de grande vitesse ferroviaire conduit les transporteurs historiques et les nouveaux entrants à concentrer logiquement leurs efforts sur les axes avec les plus forts trafics afin d'être les plus compétitifs possibles par rapport à leurs concurrents, ce qui présente certes des vertus, de réduction des coûts notamment, autrement dit de gains de productivité.

De plus, l'ouverture progressive à la concurrence renforce la segmentation entre les différents marchés ferroviaires, grande vitesse, liaisons interurbaines et liaisons régionales. Un nouvel entrant, en concentrant son offre nouvelle sur la grande vitesse, n'a pas à l'inverse de l'opérateur historique à supporter les autres liaisons ferroviaires dans toute leur diversité. Pour les transporteurs, la concurrence sur la grande vitesse limite drastiquement la géographie des Etats aux seules liaisons entre les plus grandes villes, à la vision de ce qui pourrait être qualifié d'une géographie utile voire " utilitariste » des Etats accentuant mécaniquement les inégalités et les contrastes territoriaux.

\section{Le gestionnaire du réseau}

Ce qui est vrai pour les transporteurs l'est sans doute encore plus pour le propriétaire du réseau en charge de son entretien, de sa modernisation voire de son développement. Un tuyau offrant un débit maximum, c'est-à-dire la circulation du plus grand nombre de trains possibles en une heure, permet aussi, si la demande est là, des péages élevés qui sont autant de recettes nécessaires à l'entretien et à la modernisation du réseau, voire à son développement par le remboursement des emprunts contractés pour construire les lignes nouvelles.

Pour SNCF-réseau, Paris-Lyon est le seul axe qui compte car là circulent tous les trains en provenance ou à destination du Sud-Est de la France (Cf. Encadré 1). Des projets sont actuellement en cours pour augmenter la capacité de cette liaison de 13 trains par heure à 16 voire à 19. En augmentant le débit, les économies d'échelle sont à l'œuvre. La même logique prévaut sur l'axe atlantique entre Montparnasse et la bifurcation de Courtalain (Eure-et-Loir) avec d'un côté la branche vers Bordeaux et de l'autre celle vers Le Mans et Rennes. 
En dehors de ces deux gros tuyaux et en poussant la caricature, le reste n'est que fardeau ! Ce qui est vrai pour les LGV s'applique à l'ensemble du réseau. $4 \%$ du réseau ferré national (RFN) supporte à lui seul $20 \%$ des trains-kilomètres et $40 \%$ du réseau absorbe $80 \%$ du trafic (Autorité de Régulation des Transports, 2019) ! En toute rationalité, il serait sans doute judicieux de se limiter au $4 \%$ du RFN !

D'autant plus que la construction d'une LGV nécessite des investissements lourds moyens de 15 à 25 millions d'euros du kilomètre en fonction des contraintes de la topographie et de l'urbanisation (et qui peuvent s'envoler en zone très dense sans autre solution que le tunnel). Ces infrastructures lourdes ne répondent pas directement aux lois du marché. Leur financement nécessite d'importants tours de table quels que soient les montages réalisés, en partenariat public-privé notamment. Une fraction seulement de l'investissement, variable en fonction des trafics, peut être financée par les péages, le reste devant l'être directement par de l'investissement public, en provenance de l'Etat ou des collectivités territoriales (Cf. Encadré 1 ) ...

La France a fait le choix de construire son réseau LGV par un endettement massif de son gestionnaire de réseau, RFF jusqu'en 2015 puis SNCF-réseau. La dette a explosé avec les constructions puis les mises en service des LGV Paris-Strasbourg, Rhin-Rhône, Le Mans-Rennes et Tours-Bordeaux, passant de 20 milliards d'euros en 1997 (dette héritée de la construction de la LGV Nord notamment) à 47 milliards en 2018. Ces investissements dans les lignes nouvelles se sont faits au détriment du reste du réseau, ce qui a bien été mis en évidence par deux rapports commandés par Réseau Ferré de France à l'Ecole polytechnique fédérale de Lausanne (Rivier, 2005 ; Putallaz, 2012).

Dans de telles conditions et face à un projet de ligne nouvelle ne reliant pas deux très grandes villes, le gestionnaire de réseau devrait, en suivant une logique rationnelle, ne pas le soutenir afin de ne pas dégrader encore plus ses capacités de financement au détriment du reste du réseau. II pourrait a priori le décider facilement puisqu'il occupe une situation de monopole naturel en dehors de toute concurrence (même si son réseau est en concurrence avec les autres réseaux de transport). Mais il doit tenir compte des choix politiques voulus par l'Etat et les collectivités locales, l'appétit aiguisé des bureaux d'étude et du secteur du BTP pour tous les grands travaux, sans oublier la propre appétence de ses employés, toute hiérarchie confondue, pour les exploits technologiques. Il est plus excitant de construire une LGV que d'entretenir une petite ligne...

Tenu à la fin de l'année 2007, le Grenelle de l'environnement prévoyait un plan pharaonique de doublement du réseau LGV à I'horizon 2020 pour un coût de plusieurs dizaines de milliards d'euros sans l'ombre du début d'un financement! Les projets étaient multiples et non hiérarchisés ${ }^{2}$. Face à son endettement, RFF a joué la montre en menant des études de plusieurs dizaines de millions d'euros. Plusieurs rapports et commissions ont été nécessaires (Commission Mobilités 21 en 2013, Cour des comptes en 2014, Rapport du conseil d'orientation des infrastructures en 2018) pour inverser la tendance et redonner la priorité au réseau existant, la prise de conscience étant définitive avec l'accident de Brétigny-sur-Orge en 2013 qui révèle la vétusté du réseau historique. Les projets du Grenelle sont définitivement enterrés par l'intervention du Président Macron lors de l'inauguration de la LGV Le Mans-Rennes le $1^{\text {er }}$ juillet 2017 !

\footnotetext{
2 Ils prolongent des LGV existantes vers Nice (LGV Provence-Alpes-Côte d'Azur), vers Bordeaux, Toulouse et Bayonne (LGV Sud Europe Atlantique), vers Perpignan (LGV vers Orléans et Clermont-Ferrand par le doublement de la LGV avec Lyon, cf projet POCL: Paris Orléans Clermont-Ferrand Lyon) et même un improbable projet mettant Brest et Quimper à moins de 3 heures de Paris et rapprochant Nantes et Rennes à moins de 1 heure en passant par l'aéroport de Notre-Dame des Landes (projet LNOBPL : Liaisons Nouvelles Ouest Bretagne Pays de la Loire)!
} 
Le gestionnaire du réseau ferroviaire est ainsi à la confluence d'intérêts contradictoires mais sa rationalité propre de gestionnaire de réseau devrait l'amener à ne privilégier que les seules liaisons les plus importantes.

\section{Et les territoires?}

Dans une France hyper-centralisée, la garantie de l'accès rapide à Paris est jugée essentielle par les territoires, un prérequis pour rester dans la course du développement, avec la foi chevillée au corps que le TGV apporte le développement ou en tout cas qu'il ne peut pas faire de mal, ce qui explique la participation de plus en plus lourde des Régions dans le financement des LGV, à l'exemple des Régions Bretagne ou Aquitaine impliquées massivement dans les tronçons Le Mans-Rennes (LGV BPL: Bretagne-Pays de la Loire) et Tours-Bordeaux (LGV SEA : Sud Europe Atlantique).

Les relations entre l'Etat et les collectivités, notamment les Régions et les métropoles, montrent une absence d'une vision globale de la desserte du territoire. L'Etat a de fait favorisé cette concurrence entre les territoires avec les projets de LGV issus du Grenelle de l'environnement. Sans politique claire, il n'a pas été capable d'arbitrer entre les projets, laissant le gestionnaire de réseau se dépêtrer avec les collectivités. L'objectif des collectivités étant de ne pas être en reste, II a eu les plus grandes difficultés pour les stopper face aux attentes et espoirs suscités localement.

En effet, chaque projet du Grenelle s'accompagne d'un débat public qui nécessite de gros moyens, rencontre très souvent un vif succès avec une mobilisation des différentes parties prenantes, notamment des élus, mais aussi du simple citoyen. Le débat public de la LGV POCL en fournit un bon exemple comme en témoigne la Commission Nationale du Débat Public (CNDP) dans le bilan qu'elle en tire : «Exemplaire, ce débat l'est à plusieurs titres. En premier lieu, il a suscité une participation à la hauteur des attentes des régions traversées. Plus de 14000 personnes aux réunions publiques, 220 cahiers d'acteur produits et 1200 avis ou contributions reçus par courrier ou déposés sur le site ; on comptabilise plus de 80000 connexions au site du débat (460 000 pages ont été lues), et 500 questions posées électroniquement. Évoquer cette forte mobilisation du public conduit aussi à souligner la place et le rôle importants de la presse puisque près de 1100 articles et émissions ont été consacrés au débat public " (CNDP, 2012). Ce projet POCL à plus de 14 milliards d'euros n'avait de justification économique qu'à travers le doublement de la ligne Paris-Lyon et non par les villes intermédiaires desservies. Depuis, il a été jugé préférable d'augmenter la capacité de la ligne Paris-Lyon. Beaucoup de bruit pour rien et au final une décrédibilisation des politiques publiques.

Mais alors que plus aucune LGV n'est aujourd'hui en construction, la carte isochrone des liaisons entre Paris et les plus grandes villes de France par LGV démontre un jeu à somme nulle. Elles ont été rapprochées de Paris par une réduction impressionnante des distances-temps. Deux grands perdants font exception car ils ne répondent pas au modèle classique de la LGV : la Normandie dont les trois villes principales sont à la fois trop proches de Paris, trop petites et trop éloignées les unes des autres sans former métropole, ainsi que l'Auvergne et le Limousin avec seulement deux villes de taille moyenne, Clermont-Ferrand et Limoges, situées à l'écart des axes structurants. "Observés non plus avec une loupe spatiale et temporelle mais à l'aune du temps long et de l'ensemble du réseau (...), les effets structurants sont bien là. " (Bretagnolle, 2014).

Face aux logiques des réseaux massifiés et à haut débit des transporteurs et du gestionnaire de réseau et face à l'absence de choix clairs par l'Etat sur les projets de LGV, les collectivités jouent finalement un rôle d'amortisseur territorial en défendant leurs intérêts avec l'idée qu'une LGV leur permet de ne 
pas décrocher dans la compétition territoriale aux échelles françaises et européennes. Elles le font en mettant lourdement la main à la poche en finançant directement l'infrastructure (cf. Encadré 1).

Mais elles sont désormais prises dans un engrenage infernal de financement. Depuis les années 2000, elles ont joué un rôle essentiel dans le renouveau de l'offre régionale via les réseaux TER. Cependant elles le paient cher par des conventions qui les lient avec la SNCF. Aujourd'hui, le gestionnaire du réseau, qui peine à financer la rénovation de son réseau structurant, leur demande, avec l'aval de I'Etat, de participer aussi à la rénovation des lignes secondaires.

L'ouverture à la concurrence des liaisons TER portent les mêmes dangers que celle des TGV. Les modalités de cette ouverture seront déterminantes pour l'accessibilité des territoires et les finances des Régions, autorités organisatrices des transports (AOT). Une ouverture à la concurrence liaison par liaison porte clairement le risque d'un intérêt des nouveaux entrants limité aux services les plus rentables. Les moins rentables seront à la charge de l'opérateur historique qui devra alors plus que jamais faire l'aumône auprès des AOT pour en équilibrer l'exploitation. Inversement, une ouverture à la concurrence d'un morceau du réseau peut permettre de contraindre le ou les transporteurs retenus à jouer de la péréquation entre lignes bénéficiaires et déficitaires afin de préserver une plus forte desserte de l'ensemble du réseau.

\section{Conclusion}

La rationalité des opérateurs de réseau, transporteurs et gestionnaires de réseau, les conduit à privilégier le débit, la cadence, la massification et les "gros tuyaux " entre les plus grandes villes (Frémont et al, 2020). La concurrence ne fait que renforcer cette rationalité économique du réseau: le transporteur doit préserver sa compétitivité par rapport à son concurrent et le gestionnaire de réseau celle de son réseau par rapport aux autres réseaux. Sans doute, et c'était là l'idée de la Commission européenne à logique libérale, la vertu de cette concurrence est de conduire les opérateurs à baisser leurs coûts, à gagner en compétitivité et à faire prendre conscience aux compagnies historiques, héritières des monopoles naturels, qu'elles sont mortelles... Et qu'un impératif de modernisation s'impose à elles sur les secteurs conventionnés qui ne doivent leur existence que par un apport massif d'argent public (cf. Encadré 1).

La concurrence entre les opérateurs de réseau n'est pas sans conséquence sur les territoires. Avec ou sans concurrence, la grande vitesse ferroviaire profite aux pôles les plus dynamiques, ne dynamise pas par magie les territoires en difficulté et néglige les espaces intermédiaires. La concurrence renforce ces règles et par conséquent ne fait qu'accentuer encore plus les contrastes territoriaux. Les transporteurs nouveaux entrants s'implantent là où sont les marchés et pas ailleurs. S'ils parviennent à franchir la barrière d'entrée, leurs performances deviennent supérieures à celles de l'opérateur historique national confronté à la diversité de son réseau.

Quant aux collectivités locales, principalement les Régions, elles se livrent concurrence pour obtenir " leur LGV » même si certaines sont clairement déraisonnables. En l'absence d'une vision claire de l'Etat, la concurrence pour le " tout TGV " pousse à laisser le reste du réseau ferroviaire à l'état de friche, accentuant encore le contraste entre le réseau le plus utilisé sur lequel SNCF-Réseau concentre désormais tous ces efforts et les petites lignes sans solution de financement pour leur rénovation.

Pour reprendre l'image de Pierre Veltz (2019), la France est devenue une vaste métropole par la réduction des distances-temps entre l'lle-de-France et les plus grandes aires urbaines de province. Mais la métropole France, à l'image de toutes les métropoles, est fragmentée tel un archipel dont les 
îles et leurs habitants sont très inégalement reliés. Entre l'efficacité de la concurrence à court terme appliquée aux opérateurs de réseau et un relatif équilibre entre les territoires et leurs habitants, gage de cohésion sociale et qui ne peut être construit que sur le long terme, les formes de la régulation entre les différentes parties prenantes restent à inventer.

\section{Encadré 1 : Quelques chiffres clés}

\section{Recettes des entreprises ferroviaires en 2018}

13,6 milliards d'euros dont :

- $\quad 8,4$ milliards d'euros de recettes commerciales

- 5,2 milliards d'euros de concours publics versés aux entreprises ferroviaires pour l'exploitation des services conventionnés (TER, Transilien, Intercités) et les compensations tarifaires (tarifs sociaux, militaires, etc.)

Sur les 8,4 milliards d'euros de recettes commerciales, 5,8 milliards s'expliquent par les revenus des TGV et des services internationaux (aujourd'hui principalement par TGV)

$\Rightarrow 69 \%$ des recettes commerciales hors subvention publique proviennent des TGV Source : Autorité de Régulation des Transports, Le marché français du transport ferroviaire de voyageurs 2018. https://www. autorite-transports.fr/

Recettes des gestionnaires d'infrastructure en $\mathbf{2 0 1 8}$ au titre des redevances d'infrastructure issues du transport de voyageurs

5,6 milliards d'euros dont 38\% issus des TGV domestiques et des TGV internationaux. $53,6 \%$ des péages acquittés par les EF aux gestionnaires d'infrastructure proviennent de l'activité TGV et des services internationaux.

Source : Autorité de Régulation des Transports, Le marché français du transport ferroviaire de voyageurs 2018. https://www.autorite-transports.fr/

\section{La LGV Paris-Lyon en 2017 :}

- $\quad 1 / 3$ du trafic national français en trains-kilomètres

- 240 trains par jour

- $\quad$ 7,2 millions de voyageurs en $1982 ; 44,4$ millions en 2017

Source : SNCF-réseau, 2017, modernisation de la LGV Paris-Lyon vers une ligne haute performance. https://flipbook.sncf-reseau.fr/lgv-paris-lyon-FR\#p=4

\section{Financement des LGV}

LGV Bretagne-Pays de la Loire (Le Mans-Rennes) : 3,3 milliards d'euros d'investissement dont 1,8 milliard de subventions publiques directes prises en charge à :

- $\quad 43 \%$ par RFF (devenu SNCF-Réseau)

- $\quad 28 \%$ pat l'Etat

- $\quad 28 \%$ par les collectivités locales.

Source : ERE (Eiffage) https://www.ere-lgv-bpl.com/projet/financement et http://controverses.minesparistech.fr/public/promo15/promo15 G14/www.controverses-minesparistech-

3.fr/ groupe14/exemples-emblematiques/lgv-bpl/index.html 
LGV SEA (Tours-Bordeaux) : 7,7 milliards d'euros d'investissement dont 3,9 milliards de subventions publiques directes prises en charge par SNCF-réseau, l'Etat et les collectivités.

Source : LISEA (Vinci) https://www.lisea.fr/la-lgv-sea/

\section{Références}

Antoniazzi F., Giuricin A., Tosatti R. (2019), Introducing competition in Italian high-speed railway, L'Espace Géographique, n4, p. XXX-XXX[L1].

Autorité de Régulation des Transports. (2019). Le marché français du transport ferroviaire de voyageurs 2018, tomes 1 et 2, https://www.autorite-transports.fr/

Bazin S., Beckerich C., Delaplace M., Blanquart C., Vandenbossche L. (2010). Grande vitesse ferroviaire et développement économique local : une revue de la littérature, Colloque joint ASRDLF AISRE 2010 : "Identité, Qualité et Compétitivité Territoriale. Développement économique et cohésion dans les Territoires alpins », septembre, Val d'Aoste, Italie, hal-01200665

Bretagnolle A. (2014). Les effets structurants des transports, une question d'échelles?, dossier sur Les effets structurants des infrastructures de transport, L'Espace géographique, Vol.1, tome 43, pp. 51-67.

Commission Mobilité 21. (2013). "Pour un schéma national de mobilité durable », Rapport au Ministre chargé des transports, de la mer et de la pêche, 89p. https://www.ecologiquesolidaire.gouv.fr/priorisation-des-nouvelles-infrastructures-transport-commission-mobilite-21

CNDP. (2012). Bilan du débat public sur le projet de Ligne à Grande Vitesse PARIS / ORLÉANS / CLERMONT-FERRAND / LYON du 3 octobre 2011 au 31 janvier 2012 dressé par le Président de la Commission nationale du débat public, 20 mars, 20p.

https://cpdp.debatpublic.fr/cpdp-Igv-pocl/ACTUALITE/ACTUALITE VIEW.HTM

Conseil d'orientation des infrastructures. (2018). « Mobilités du quotidien. Répondre aux urgences et préparer l'avenir ", Rapport à Madame la Ministre en charge des transports, 19p.

https://www.ecologique-solidaire.gouv.fr/sites/default/files/2018.02.01 rapport coi.pdf

Cour des Comptes. (2014). " la grande vitesse ferroviaire : un modèle porté au-delà de sa pertinence ", 173p. www.ccomptes.fr

Delaplace M. (2012). "Pourquoi les «effets» tgv sont-ils différents selon les territoires? L'hétérogénéité au cœur du triptyque «Innovations, territoires et stratégies ». Recherche Transports et Sécurité, $\mathrm{n}^{\circ} 28$, p. 290-302.

Frémont A., Pons A. (2020). Quel rôle pour les entreprises de réseaux dans l'aménagement du territoire ?, in sous la direction de Cordobès S., Desjardin X., Vanier M., Actes du colloque de Cerisy sur la nouvelle pensée aménagiste, Berger Levrault, à paraitre.

Pini G. (1992). L'interaction spatiale, in Antoine Bailly, Robert Ferras, Denise Pumain (ss la dir.) Encyclopédie de géographie, Paris, Economica, pp.557-576.

Plassard F. (1992). Les réseaux de transports et de communication, in Antoine Bailly, Robert Ferras, Denise Pumain (ss la dir.) Encyclopédie de géographie, Paris, Economica, pp.533-556. 
Putallaz Y., Tzieropoulos P. (2012). « Audits EPFL revisités. Synthèse des travaux du groupe d'études. Audit sur l'état du réseau. (Audit Rivier) ", Ecole Polytechnique Fédérale de Lausanne, 39p. http://www.clac-info.fr/sites/default/files/Rapport EPFL sept 2012.pdf

Quinet E. (2013). Evaluation socioéconomique des investissements publics, Rapports et documents, Commissariat général à la stratégie et à la prospective, Paris, 349p.

Rivier R., Putallaz Y. (2005). « Audit sur l'état du réseau ferré national français. Rapport principal et rapports détaillés sectoriels. Ecole Polytechnique Fédérale de Lausanne.

Veltz P. (2019). La France des territoires, défis et promesses, L'Aube. 